Research Paper

\title{
Placenta-Derived Mesenchymal Stem Cells Reduce the Interleukin-5 Level Experimentally in Children with Asthma
}

\author{
Sheng-Chieh Lin¹, 2, 3, Yih-Mei Liou ${ }^{4}$, Thai-Yen Ling 5 , Ya-Hui Chuang4,6® ${ }^{\circledR}$, Bor-Luen Chiang ${ }^{3}$ \\ 1. Department of Pediatrics, Shuang Ho Hospital, Taipei Medical University, Taipei, Taiwan. \\ 2. Department of Pediatrics, School of Medicine, College of Medicine, Taipei Medical University, Taipei, Taiwan. \\ 3. Graduate Institute of Clinical Medicine, College of Medicine, National Taiwan University, Taipei, Taiwan. \\ 4. Department of Clinical Laboratory Sciences and Medical Biotechnology, College of Medicine, National Taiwan University, Taipei, Taiwan. \\ 5. Department of Pharmacology, College of Medicine, National Taiwan University, Taipei, Taiwan. \\ 6. Department of Laboratory Medicine, National Taiwan University Hospital, College of Medicine, National Taiwan University, Taipei, Taiwan. \\ $\square$ Corresponding author: Ya-Hui Chuang, Ph. D., Department of Clinical Laboratory Sciences and Medical Biotechnology, College of Medicine, National \\ Taiwan University. No. 1, Chang-Te Street, 10016, Taipei, Taiwan, TEL: 886-2-2312-3456 Ext. 66906, FAX: 886-2-2371-1574, E-mail: yahuichuang@ntu.edu.tw \\ (c) The author(s). This is an open access article distributed under the terms of the Creative Commons Attribution License (https://creativecommons.org/licenses/by/4.0/). \\ See http://ivyspring.com/terms for full terms and conditions.
}

Received: 2019.01.28; Accepted: 2019.06.21; Published: 2019.09.20

\begin{abstract}
Background: Mesenchymal stem cells (MSCs) have been investigated as a new treatment option for various diseases in recent years. However, the role of placenta-derived MSCs in children with asthma remains unclear. We assessed the effect of placenta-derived MSCs on T cell immune responses and cytokine IL-5 levels according to cultures in children with and without asthma.

Study design: We enrolled children with and without asthma and recorded asthma symptom scores in the asthma group. Blood samples from children were collected to isolate peripheral blood mononuclear cells (PBMCs) and determine the total lgE level. The PBMCs were cultured in vitro with or without MSCs after stimulation with human anti-CD3 and anti-CD28 antibodies $(0.5 \mu \mathrm{g} / \mathrm{mL})$ to evaluate the effect of placenta-derived MSCs. Flow cytometry was performed to detect the activation and proliferation of CD4+ and CD8+ T cells. Pre- and post-culture IL-5 levels were measured in all samples.

Results: The percentages of activation and proliferation among CD4+ and CD8+ T cells after coculture with MSCs were significantly lower in the asthma group $(P<0.05)$. IL-5 levels differed significantly between the PBMC culture and PBMC + MSC $(P+S)$ coculture in the asthma group $(P<$ $0.05)$. IL-5 levels differed significantly between the PBMC culture and P+S coculture in both the lower $(P<0.05)$ and higher $(P<0.0005)$ lgE asthma subgroups. IL-5 levels were also decreased in children with all severities of asthma $(P<0.05)$.

Conclusions: Placenta-derived MSCs exerted an anti-IL-5 effect and reduced the IL-5 level in culture in different subgroups of children with asthma.
\end{abstract}

Key words: asthma, allergy, mesenchymal stem cell, IL-5, CD4, CD8.

\section{Introduction}

Asthma is a common respiratory tract disease that manifests as an allergic and inflammatory process in children. Although several factors related to asthma are understood, its precise cause remains unknown. The complex relationships among genetic, environmental, pharmacological, and immunological factors in this disease still require further investigation [1]. Asthma is relatively common, and the pathophysiology of asthma is characterized by injury, inflammation, and eventually the remodeling of airways [2]. For a long time, asthma was regarded as a chronic type $2 \mathrm{~T}$ helper (Th2) cell-driven disease with eosinophilic airway inflammation [3]. Eosinophils develop from hematopoietic CD34+ 
progenitor cells, and interleukin (IL)-5 is a key cytokine involved in the differentiation, proliferation, and survival of eosinophils [4]. IL-5 is mostly secreted by allergen-reactive $\mathrm{T}$ cells, mast cells, and eosinophils [5]. A study reported that CD34+/IL-5+ cells could be found in patients with asthma [4]. Notably, IL-5 plays a crucial role in asthma. In recent years, stem cell transplantation has been evaluated as a disease therapy. Hematopoietic stem cell transplantation is a life-saving treatment for severe combined immunodeficiency even when an HLA-identical donor is not available [6]. Tan et al. [7] reported that placenta-derived mesenchymal stem cells (MSCs) and methylprednisolone exerted a significant effect on the recovery of neurological function with neuroprotective effects in an animal model. MSCs can undergo proliferation and multiple differentiations, possess strong potential for tissue regeneration, and exert anti-inflammatory and immunomodulatory effects $[2,8,9,10,11]$. MSCs in bone marrow can differentiate into cells that constitute multiple nonhematopoietic organs [8]. Many studies have examined the use of stem cell therapy in various diseases such as acute lung injury [12], acute pancreatitis [13], and hepatic failure [14]. In allergic rhinitis in adults, bone marrow-derived MSCs and induced pluripotent stem cell-derived MSCs (iPSC-MSCs) significantly suppress Th2 cells, promote regulatory $\mathrm{T}$ (Treg) cell responses, and inhibit lymphocyte proliferation in peripheral blood mononuclear cells (PBMCs) [15]. However, Desai et al. [16] reported that MSCs presented allergens to CD4+ $\mathrm{T}$ cells, thus causing an increase in the production of inflammatory cytokines and the proliferation of lymphocytes in allergic rhinitis in adults. Nevertheless, no study has focused on the therapeutic effects of MSCs on asthma in children. Intravenous MSCs transplantation in murine asthma models improved the pathological features of asthma, some of which include decreased collagen deposition and inflammatory infiltration around the airway $[9,17,18,19]$, blunt airway hyper-responsiveness $[19,20,21]$, improved pulmonary histological scores $[17,22,23]$, and remodeling prevention $[24,25,26]$. MSCs and serelaxin exerted a synergistic effect to prevent airway fibrosis in an experimental model of chronic allergic airway disease [27]. In a cat model of asthma, MSC-treated animals exhibited decreased airway inflammation with significantly lower bronchial wall thickening scores and lung attenuation on computed tomography images 8 months after the study [24]. MSC-treated asthmatic cats also exhibited significantly decreased airway inflammation, parenchymal changes, airway hyper-responsiveness, and airway remodeling [25]. Repeated administration of allogeneic adipose-derived MSCs exerted a delayed effect in preventing airway remodeling in asthmatic cats [25].

Conventionally, glucocorticoids and bronchodilators are used for the treatment of asthma. However, some children whose asthma cannot be easily controlled with the use of glucocorticoids may require alternative treatment. Promising therapeutic methods that showed favorable outcomes in animal models of asthma should be converted into clinical practice in humans. However, the exact mechanism of function of placenta-derived MSCs in children remains unclear and requires further evaluation. This study evaluated the effect of placenta-derived MSCs on the activation and proliferation of CD4+ T cells and CD8+ T cells in children with and without asthma. In addition, we evaluated the mechanisms through which MSCs exerted an anti-IL-5 effect and attempted to understand the potential of MSC therapy in children with asthma.

\section{Materials and Methods}

\section{Participants and data collection}

In this study, we enrolled children who had been diagnosed with asthma and children without asthma. Inclusion criteria for the asthma group were based on the Global Initiative for Asthma guidelines [26]. Children who had acute asthma exacerbation and did not use steroids within 1 week before the study were included in the asthma group. Children without asthma were included in the nonasthma group. Children who had parasitic infections or hepatic disorders were excluded. This study was approved by the Institutional Review Board of Taipei Medical University and was conducted in accordance with the Declaration of Helsinki. After obtaining parental written informed consent, we collected a 5-mL sample of peripheral blood and recorded children's age and sex. We enrolled 68 children ( 28 girls and 40 boys), 45 and 23 of whom were included in the asthma and nonasthma groups, respectively. The sex ratio was 0.67:1 (18 girls and 27 boys) and 0.77:1 (10 girls and 13 boys) in the asthma and nonasthma groups, respectively. At the time of the study, all children were between the ages of 2 and 12 years, with a mean age of 6 years $(6.00 \pm 0.26)$. The average ages of children in the asthma and nonasthma groups were 5.88 years $(5.88 \pm 0.32)$ and 6.25 years $(6.25 \pm 0.44)$, respectively. No statistically significant intergroup age difference was observed $(P=0.50)$. The demographics of the children are listed in Table 1.

\section{Asthma symptom score}

The asthma symptom score was determined for children with asthma. The score ranged from 0 to 16 
points for the following symptoms. Nighttime cough: $0=$ absent, $1=$ mild (present but not disturbing sleep), $2=$ moderate (awake once because of cough), $3=$ severe (awake more than once because of cough), and $4=$ extremely severe (insomnia throughout the night). Shortness of breath early in the morning: $0=$ absent, 1 $=$ mild (occasional and no medication required), $2=$ moderate (occasional and medication required), $3=$ severe (frequent and medication required), and $4=$ extremely severe (persistent and multiple doses of medication required). Daytime wheezing or dyspnea: $0=$ absent, $1=$ mild (occasional wheezing), 2 = moderate (occasional wheezing and dyspnea but not disruptive of daily activities), 3 = severe (persistent and disruptive of daily activities), and $4=$ extremely severe (completely prevents daily activities). Daytime cough: 0 = absent, $1=$ mild (occasional but not disruptive of daily activities), 2 = moderate (frequent but not disruptive of daily activities), 3 = severe (frequent and disruptive of daily activities), and $4=$ extremely severe (persistent) [26,28]. Patients with total asthma symptom scores from 1 to 4 , from 5 to 8 , from 9 to 12, and from 13 to 16 were defined as having mild, moderate, severe, and extremely severe asthma symptoms, respectively [28].

\section{Subgroup classification}

We divided the children into subgroups to evaluate the effect of stem cells on different groups. The children with asthma who had total IgE levels of $<200 \mathrm{KU} / \mathrm{L}$ and $>200 \mathrm{KU} / \mathrm{L}$ were categorized into lower $(\mathrm{n}=15)$ and higher $\operatorname{IgE}(\mathrm{n}=30)$ asthma subgroups, respectively. The sex ratios in the lower and higher IgE asthma subgroups were 0.88:1 (7 girls and 8 boys) and 0.58:1 (11 girls and 19 boys), respectively. The average ages of children in the lower and higher IgE asthma subgroups were 5.79 years $(5.79 \pm 0.55)$ and 5.92 years (5.92 \pm 0.41$)$, respectively. No statistically significant intersubgroup age difference was noted $(P=0.84)$. The demographics of the participants are listed in Table 1.

Table 1. Participant Demographics

\begin{tabular}{ll}
\hline Number of children (total) & 68 \\
Ratio of children without asthma to those with asthma & $23: 45$ \\
Ratio of lower to higher IgE asthma & $15: 30$ \\
Ratio of girls to boys (total) & $28: 40$ \\
Nonasthma (girls:boys) & $10: 13$ \\
Asthma (girls:boys) & $18: 27$ \\
Lower IgE asthma (girls:boys) & $7: 8$ \\
Higher IgE asthma (girls:boys) & $11: 19$ \\
Mean age (total) & $6.00 \pm 0.26$ \\
Age ratio of nonasthma to asthma & $6.25 \pm 0.44: 5.88 \pm 0.32$ \\
Age ratio of lower to higher IgE asthma & $5.79 \pm 0.55: 5.92 \pm 0.41$ \\
Mean asthma symptom score (asthma) & $7.76 \pm 0.56$ \\
Asthma symptom score ratio of lower to higher IgE asthma & $7.47 \pm 0.8: 7.9 \pm 0.74$ \\
\hline
\end{tabular}

\section{Molecular methods}

\section{PBMC isolation}

We used density gradient centrifugation (Histopaque; Sigma Aldrich, St. Louis, MO, USA) to isolate PBMCs from heparinized venous blood. Initially, we depleted plasma through centrifugation (400 $\times g$ for $20 \mathrm{~min}$ ). Residual blood cells were mixed with an equal volume of RPMI 1640 medium. Subsequently, Histopaque was added to the bottom of the centrifuge tube. The blood-RPMI 1640 mixture (the volume ratio of cells to Histopaque was 2:1) was slowly layered on the Histopaque solution. Next, the centrifuge tube was centrifuged at $400 \times g$ at $20^{\circ} \mathrm{C}$ for $30 \mathrm{~min}$. By using a sterile pipette, we transferred the mononuclear cell layer to a second centrifuge tube. The cells were washed and collected after adding a 2-fold excess of Hanks' Balanced Salt Solution, and then the PBMCs were prepared for cell culture.

\section{MSCs isolation}

We isolated placenta-derived MSCs from the choriodecidual membrane of human placentas [29]. The choriodecidual tissues donated by women who had undergone cesarean sections were digested using SMEM medium supplemented with $0.5 \mathrm{mg} / \mathrm{mL}$ protease, $0.5 \mathrm{mg} / \mathrm{mL}$ collagenase $\mathrm{B}$, and $1 \mathrm{mg} / \mathrm{mL}$ DNase I at $4{ }^{\circ} \mathrm{C}$ overnight and filtered through a $100-\mu \mathrm{m}$ nylon membrane. After centrifugation, cells were collected and subsequently resuspended in culture medium (MCDB201 supplemented with 1\% insulin transferrin selenium, $10 \mathrm{ng} / \mathrm{mL}$ epidermal growth factor, and $1 \%$ penicillin/streptomycin). Next, the cells were plated in culture dishes coated with human collagen type IV. The adherent cells were kept in the culture medium; every 3 or 4 days, the medium was changed and the nonadherent cells were removed. Cells that were demonstrated to be placenta-derived MSCs were cultured in a serum-free medium, which displayed fibroblast-like morphology after attachment with a positive expression for CD29, CD44, CD73, CD90 and negative expression for CD14, CD34, CD45, and HLA-DR. In vitro assays of these cells showed positive signals for adipogenic, chondrogenic, and osteogenic differentiation when stained with Alcian Blue, Alizarin Red S, and Oil Red O, respectively [29].

\section{In vitro culture of PBMCs with or without MSCs}

We cultured PBMCs at $2 \times 10^{5}$ cells $/ \mathrm{mL}$ in a RPMI 1640 medium in 96-well plates. These cells were stimulated with human anti-CD3 $(0.5 \mu \mathrm{g} / \mathrm{mL}$; Biolegend, San Diego, CA, USA) and human anti-CD28 antibodies $(0.5 \mu \mathrm{g} / \mathrm{mL}$; Biolegend $)$ and cocultured with and without $10 \%$ placenta-derived 
MSCs (MSC:PBMC $=1: 10$ ). In the asthma group, we also used different concentrations of placenta-derived MSCs (MSC: PBMC = 1:5 and 1:20). After 72 hours; cell supernatants were harvested and frozen at $-80^{\circ} \mathrm{C}$.

\section{Flow cytometry to detect cell activation and proliferation}

The presence of the surface marker CD25 was detected in cultured cells to evaluate cell activation. These cells were stained with the fluorescent anti-CD25 antibody for $30 \mathrm{~min}$ at $4{ }^{\circ} \mathrm{C}$ and fixed in $1 \%$ paraformaldehyde. In addition, to evaluate cell proliferation, cells were stained with $5-\mu \mathrm{M}$ carboxyfluorescein succinimidyl ester (CFSE) in phosphate-buffered saline (PBS) at room temperature for $5 \mathrm{~min}$ and then washed with 10 volumes of media with $10 \%$ PBS for $5 \mathrm{~min}$ before culturing. After culturing, all cells were examined for the presence of surface antigens by using flow cytometry.

\section{Detection of total IgE}

We analyzed the total plasma IgE level by using an ImmunoCAP 250 analyzer (Phadia, Uppsala, Sweden). We added $40 \mu \mathrm{L}$ of plasma to ImmunoCAP (Phadia) and incubated this mixture. The ImmunoCAP tube was then washed with a washing solution. Enzyme-labeled anti-IgE (conjugate against total IgE) was added to the ImmunoCAP and washed again. The bound total IgE was quantified using a fluorescent substrate. The ImmunoCAP testing system is highly automated (ImmunoCAP 250, Phadia, Uppsala, Sweden), and the process required $1.5 \mathrm{~h}$ in total.

\section{Enzyme-linked immunosorbent assay of the cytokine IL-5}

We determined the IL-5 level by performing enzyme-linked immunosorbent assays with commercial human cytokine kits (R\&D, Minneapolis, MN, USA). We measured the absorbance at 450 and $570 \mathrm{~nm}$ and subtracted the absorbance value obtained at $570 \mathrm{~nm}$ from that obtained at $450 \mathrm{~nm}$ to detect the IL-5 level. The absorbance was measured using the SpectraMax M5 multidetection microplate reader (Molecular Devices, Sunnyvale, CA, USA).

\section{Statistics}

All data analyses were performed using STATA 12. Using the case-control method and paired $t$-tests, we evaluated the rates of activation and proliferation among CD4+ T cells and CD8+ T cells and compared them between the PBMC culture and PBMC + MSC $(\mathrm{P}+\mathrm{S})$ coculture in the asthma and nonasthma groups. We determined IgE and IL-5 levels and compared their values between the PBMC culture and P+S coculture in the asthma and nonasthma groups by using the case-control method and paired $t$-tests. The IL-5 levels in the subgroups were analyzed using one-way analysis of variance (ANOVA) followed by Bonferroni's analysis. A $P$ value of $<0.05$ was considered significant.

\section{Results}

\section{Analysis of total serum IgE}

The total IgE level was significantly higher in the asthma group than in the nonasthma group $(P<0.005$, Figure 1A). The total IgE level significantly differed between the lower and higher IgE asthma subgroups $(P<0.0005$, Figure 1B).

(A)

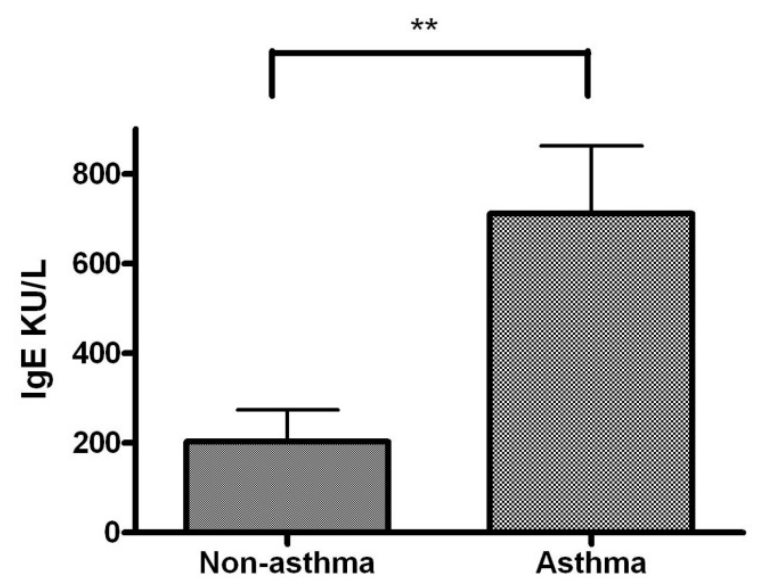

(B)

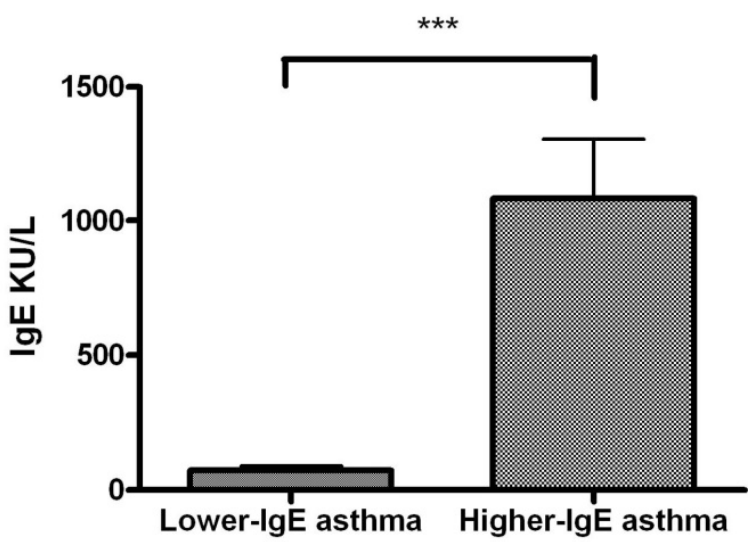

Figure 1. (A) Comparison of total lgE level between the children without asthma ( $\mathrm{n}$ $=23)$ and children with asthma $(n=45)$. (B) Comparison of the total IgE level between lower and higher lgE asthma subgroups. * indicates $P<0.05$; ** indicates $P<$ 0.005 ; *** indicates $P<0.0005$.

\section{Analysis of activation and proliferation of CD4+ and CD8+ $T$ cells in PBMC culture and $\mathbf{P}+\mathrm{S}$ coculture}

The rate of the activation of $\mathrm{CD} 4+\mathrm{T}$ cells significantly differed between the PBMC culture and 
$\mathrm{P}+\mathrm{S}$ coculture in the asthma group $(P<0.0005)$ but not in the children without asthma (Figure 2A). The rate of the proliferation of CD4+ $\mathrm{T}$ cells significantly differed between the PBMC culture and $\mathrm{P}+\mathrm{S}$ coculture in both the asthma $(P<0.0005)$ and nonasthma groups $(P<0.05$, Figure 2B). The rate of activation of CD8+ T cells significantly differed between the PBMC culture and $\mathrm{P}+\mathrm{S}$ coculture in the asthma group $(P<0.0005)$ but not in the nonasthma group (Figure $2 \mathrm{C}$ ). The rate of the proliferation of CD8 $+\mathrm{T}$ cells significantly

\section{(A)}
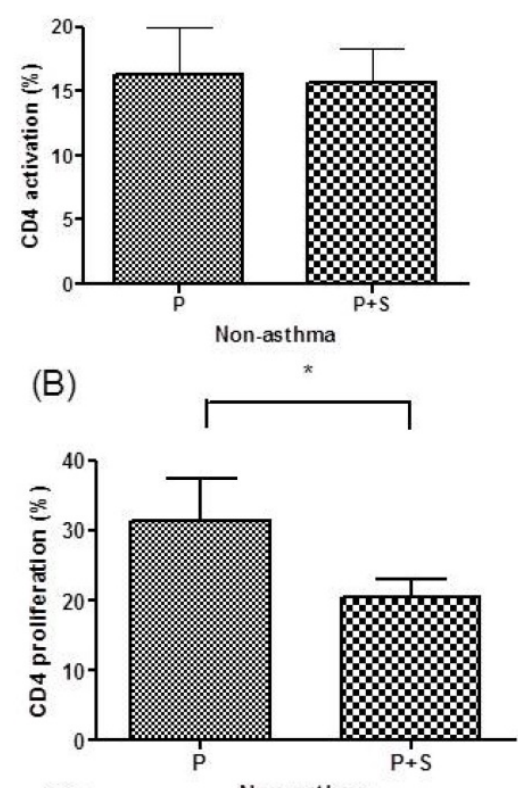

(C)
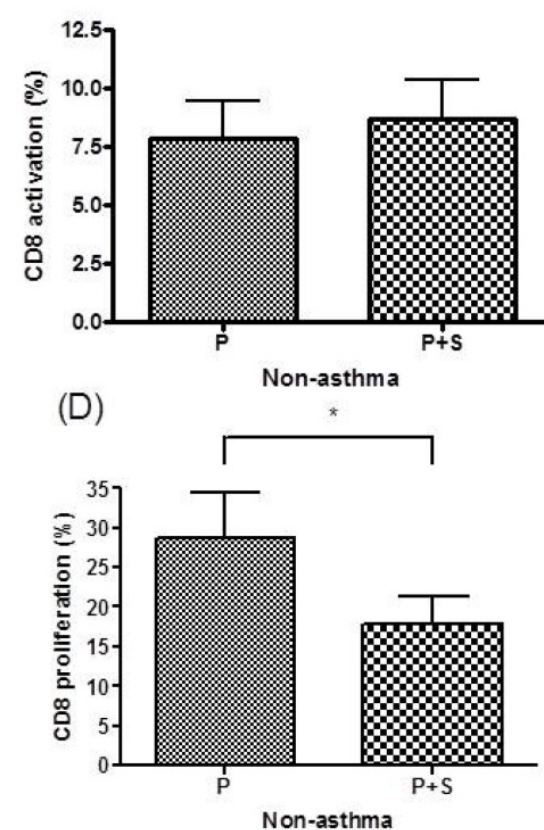

differed between the PBMC culture and $\mathrm{P}+\mathrm{S}$ coculture in both the asthma $(P<0.0005)$ and nonasthma groups $(P<0.05$, Figure 2D).

\section{Analysis of the IL-5 level between the PBMC culture and $\mathbf{P + S}$ coculture}

IL-5 levels differed significantly between the PBMC culture and $\mathrm{P}+\mathrm{S}$ coculture in the nonasthma $(P$ $<0.05$, Figure $3 \mathrm{~A})$ and asthma groups $(P<0.05$, Figure 3B).
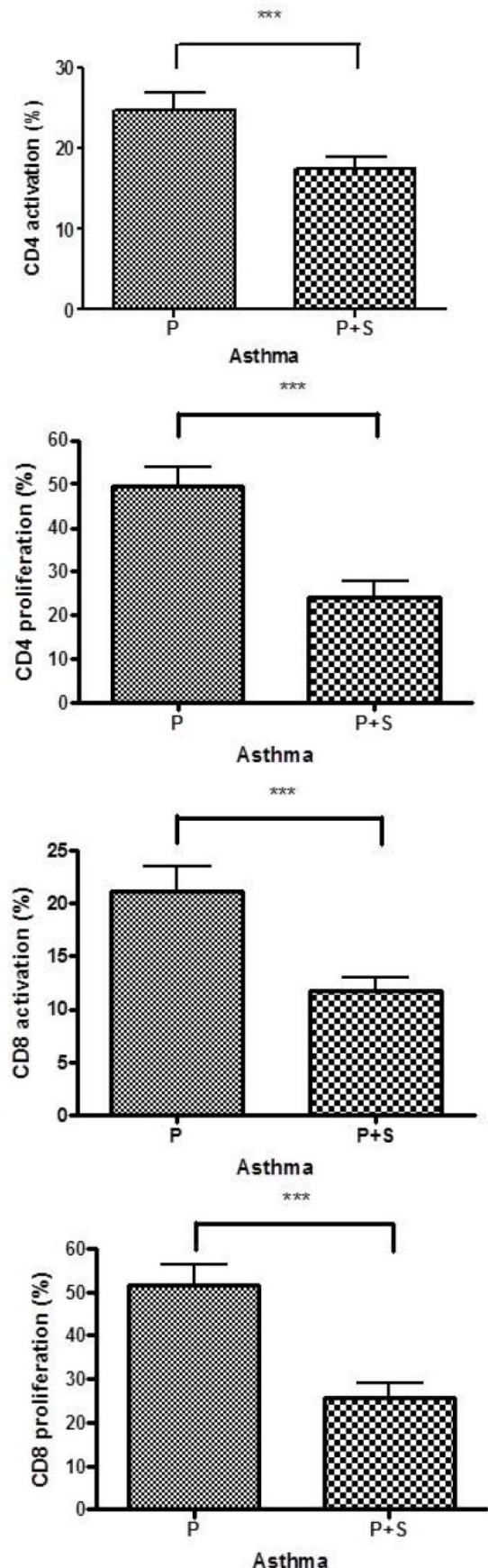

Figure 2. (A) Percentages of CD4+ T cell activation in the PBMCs $(P)$ culture and PBMC + MSC (P+S) coculture in the nonasthma and asthma groups. (B) Percentages of CD4+ $T$ cell proliferation in the $P$ culture and $P+S$ coculture in the nonasthma and asthma groups. $(C)$ Percentages of $C D 8+T$ cell activation in the $P$ culture and $P+S$ coculture in the nonasthma and asthma groups. (D) Percentages of CD8+ T cell proliferation in the $\mathrm{P}$ culture and $\mathrm{P}+\mathrm{S}$ coculture in the nonasthma and asthma groups. $*$ indicates $P<0.05$; $* *$ indicates $P<0.005$; *** indicates $P<0.0005$. 


\section{Clinical severity in children with asthma}

The asthma symptom scores of the asthma group, lower $\operatorname{IgE}$ asthma subgroup, and higher $\operatorname{IgE}$ asthma subgroup was $7.76 \pm 0.56,7.47 \pm 0.8$, and $7.9 \pm$ 0.74 , respectively. The asthma symptom score did not differ significantly between the lower and higher $\operatorname{IgE}$ asthma subgroups $(P=0.69$, Table 1$)$.

(A)

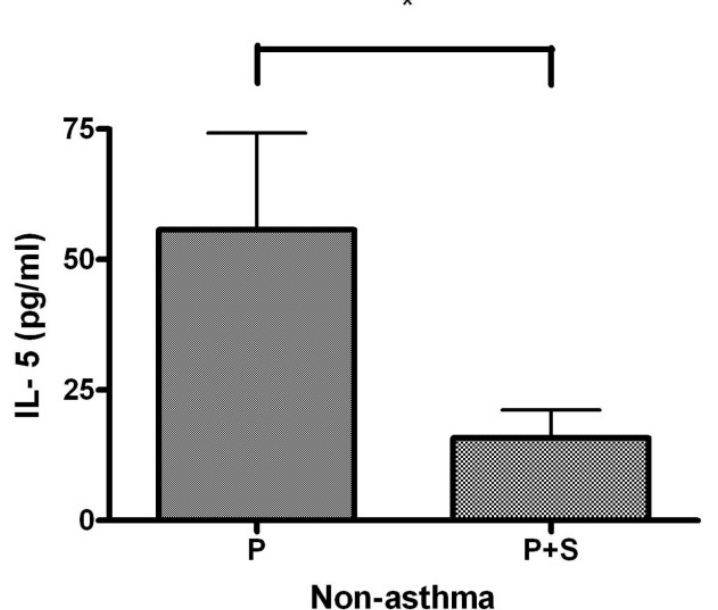

(B)

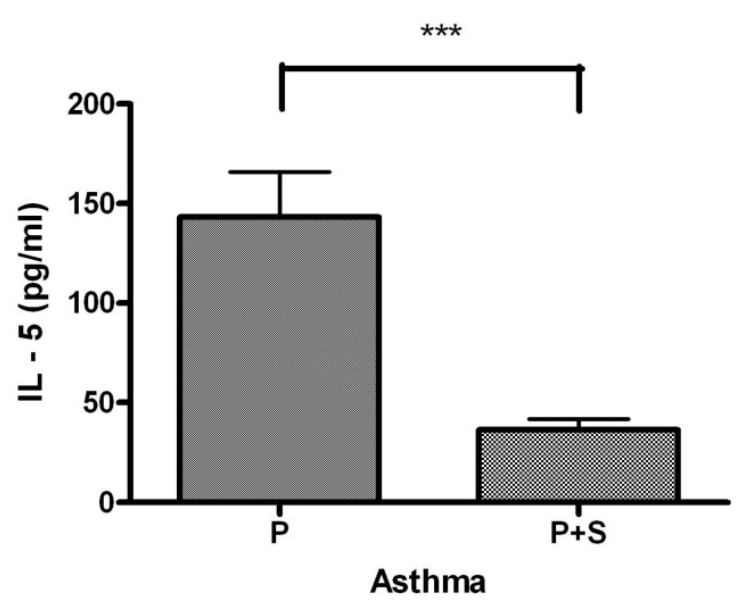

Figure 3. (A) Comparison of IL-5 levels between the PBMC (P) culture and PBMC + MSC $(P+S)$ coculture in children without asthma. (B) Comparison of IL-5 levels between the $P$ culture and $P+S$ coculture in children with asthma. * indicates $P<0.05$; ** indicates $P<0.005$; *** indicates $P<0.0005$.

\section{Comparison of IL-5 levels between the PBMC culture and $\mathbf{P}+\mathbf{S}$ coculture in the asthma subgroups}

IL-5 levels significantly differed between the PBMC culture and P+S coculture in both the lower and higher IgE asthma subgroups $(P<0.05$, Figure $4 \mathrm{~A})$. The results of one-way ANOVA revealed that IL-5 levels differed significantly between the PBMC culture and $\mathrm{P}+\mathrm{S}$ coculture (different MSC concentrations) in the asthma group $(F=13.59$, degrees of freedom $=142, P<0.0005$, Figure 4B). IL-5 levels differed significantly between the PBMC and $\mathrm{P}+\mathrm{S}$ coculture and among the children with asthma who had mild $(P<0.05)$, moderate $(P<0.05)$, severe $(P<0.05)$, and extremely severe $(P<0.05)$ asthma symptom scores (Figure $4 \mathrm{C}$ ).

(A)

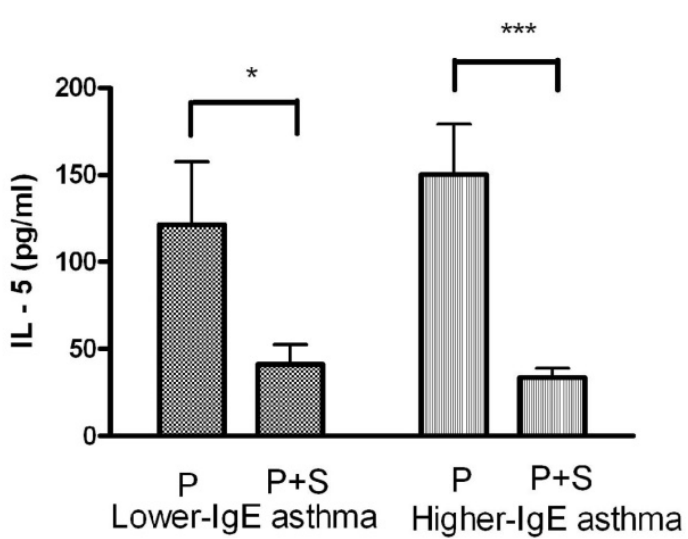

(B)

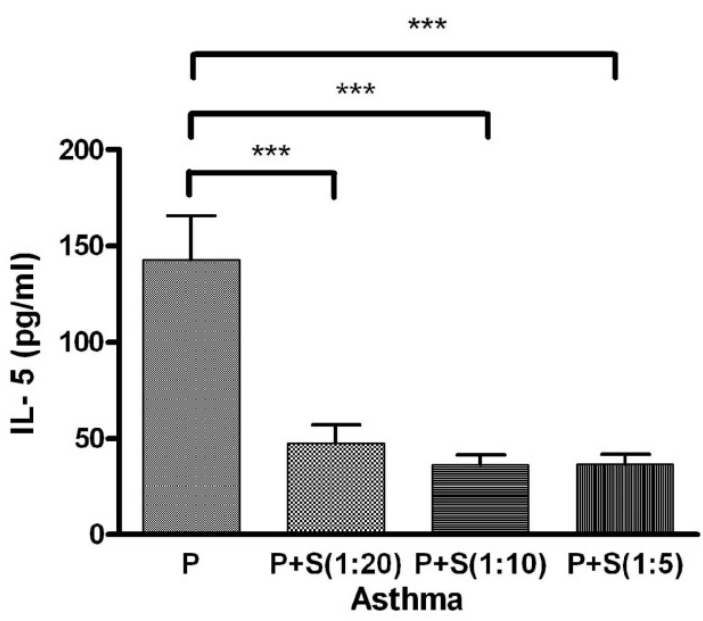

(C)

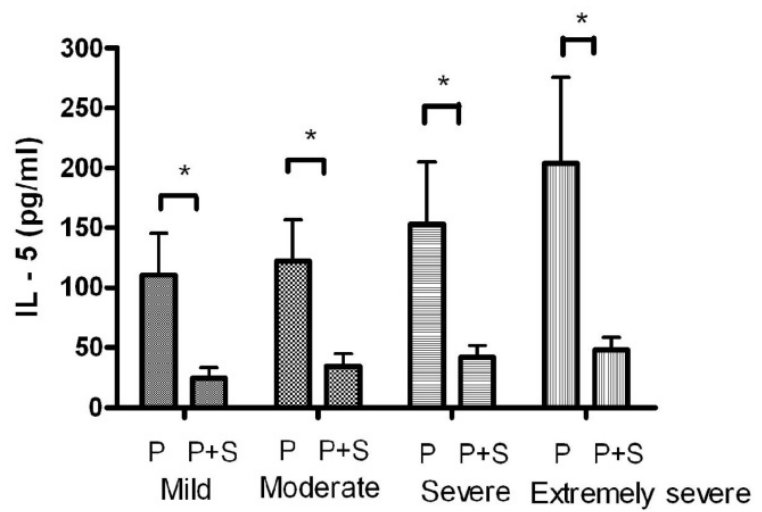

Figure 4. (A) Comparison of the IL-5 level between the PBMC (P) culture and $\mathrm{PBMC}+\mathrm{MSC}(\mathrm{P}+\mathrm{S})$ coculture in lower and higher IgE asthma subgroups. (B) Comparison of the IL-5 level between the $P$ culture and $P+S$ coculture in children with asthma. (C) Comparison of the IL-5 level between the $P$ culture and $P+S$ coculture in children with mild, moderate, severe, and extremely severe asthma symptom scores. * indicates $P<0.05$; ** indicates $P<0.005$; *** indicates $P<0.0005$. 


\section{Discussion}

Several studies have examined the mechanisms of MSCs in animal models, but none have focused on children with asthma. In murine models of asthma, MSCs were shown to be immunomodulatory and normalize the balance of type $1 \mathrm{~T}$ helper (Th1) cells and Th2 cells $[19,21,23]$. The administration of MSCs in the murine models of asthma changes the phenotype of $\mathrm{T}$ lymphocytes from Th2 to Th1 cells $[18,21]$. MSC therapy reduced airway eosinophilia in rodent models, regardless of the source of MSCs or the method of administration [17,18,19,22,24]. Notably, the percentage of eosinophils was observed to decrease to its normal level several months after MSC treatment in cat models [25].

Murine studies have reported reductions in inflammatory cells and inflammatory mediators, such as tumor necrosis factor-a and IL-6, in both bronchoalveolar lavage fluid and serum after MSC transplantation [9]. MSCs may also promote immune tolerance through the proliferation of Treg cells and the secretion of regulatory cytokines such as IL-10 in asthmatic mice [19,23]. Transforming growth factor-beta (TGF- $\beta$ ) is a type of cytokine that causes airway inflammation and airway remodeling [30,31], and MSCs can suppress TGF- $\beta$ by inhibiting alveolar macrophage polarization through the TGF- $\beta$ signaling pathway [32]. The overexpression of p-Akt was suppressed in the lung tissues of asthmatic rats after MSC transplantation [9]. MSCs can prevent inflammatory infiltration around the airway deposition of collagen and airway remodeling in a rat model of chronic asthma by disrupting the PI3K/AKT signaling pathway [9]. In a small number of adult patients with allergy-induced asthma, MSCs significantly reduced the interferon- $\gamma$ level and increased the IL-10 level [33].

We postulated that in children with asthma, the mechanism of MSCs would involve the modulation of $\mathrm{T}$ cell immune responses and cytokine IL-5. In the present study, we evaluated the effects of MSCs on CD4+ $\mathrm{T}$ cells and CD8+ $\mathrm{T}$ cells by using an in vitro culture of PBMCs and a coculture of PBMCs with MSCs. Regarding $\mathrm{T}$ helper cells, we discovered that the activation of CD4+ T cells was significantly lower in the children with asthma treated with MSCs than in those not treated with MSCs. However, no significant differences were observed among the children without asthma between those who received and who did not receive MSC treatment. MSCs appeared to suppress the activation of $\mathrm{T}$ helper cells in PBMCs cultured in vitro after stimulation with antihuman CD3 and CD28 antibodies in the children with asthma but not in the children without asthma. In our study, we also found that $\mathrm{CD} 4+\mathrm{T}$ cell proliferation was significantly lower in children with asthma who received MSC treatment than in those who did not receive MSC treatment. Significant differences were also noted in children without asthma. MSCs could suppress the proliferation of CD4+ T cells in PBMCs cultured in vitro after stimulation with antihuman CD3 and CD28 antibodies in both children with and without asthma. Regarding CD8 $+\mathrm{T}$ cells, we discovered that the rate of activation of CD8+ T cells was significantly lower in the children with asthma who received MSC treatment than in those who did not receive MSC treatment. However, no significant differences were noted between the children without asthma who received or did not receive MSC treatment. In addition, we observed that the proliferation of CD8+ $\mathrm{T}$ cells was significantly lower in children with asthma who received MSC treatment than in those who did not receive MSC treatment. Significant differences were also noted in children without asthma. MSCs could suppress the proliferation of CD8+ T cells in PBMCs cultured in vitro after stimulation with antihuman $\mathrm{CD} 3$ and CD28 antibodies in both the children with and without asthma. MSCs appeared to have a suppressive function that affected CD4+ and CD8+T cell activation and proliferation in children with asthma. Furthermore, we evaluated the effect of MSCs on IL-5 levels by using an in vitro culture of PBMCs cocultured with MSCs. IL-5 levels were significantly lower in the $\mathrm{P}+\mathrm{S}$ coculture both in the children with and without asthma. MSCs appeared to exert an anti-IL-5 effect on the in vitro culture in both the children with and without asthma. Therefore, to evaluate the anti-IL-5 effect of MSCs in children with asthma under different conditions, we divided them into subgroups. We used different concentrations of MSCs for co-culturing with PBMCs. We observed that $5 \%, 10 \%$, and $20 \%$ of MSCs cocultured with PBMCs exerted an anti-IL-5 effect. Notably, MSCs demonstrated a strong anti-IL-5 effect at different concentrations. In addition, we observed that the P+S coculture significantly reduced IL-5 levels in all of the children with asthma who had mild, moderate, severe, or extremely severe asthma symptom scores. This finding indicates that the MSCs exerted an anti-IL-5 effect in children with asthma with varying clinical severities. A clinical therapeutic study reported that the anti-IL-5 monoclonal antibody $(\mathrm{mAb})$ inhibited eosinophil development and reduced the presentation of clinical asthma symptoms [34]. Molfino et al. [35] reported that targeting IL-5 and its receptor is a rational therapy. The Food and Drug Administration of the United States has approved mepolizumab and reslizumab as new anti-IL-5 therapeutics for the treatment of patients with severe 
eosinophilic asthma [36]. Mepolizumab is a type of humanized anti-IL-5 mAb that has been widely studied. Mepolizumab was found to improve FEV1 and reduce the number of eosinophils in the sputum and blood during asthma treatment [37].

Nonetheless, our study was performed on a small number of children and only focused on IL-5. Despite this limitation, the results revealed significant differences after MSC coculture. We will conduct further studies in the future to evaluate a range of Th2-associated cytokines (e.g., IL-4 and IL-13) and also test current concepts regarding the addition of MSCs with different $\mathrm{T}$ cell subsets, such as Th9 (e.g., IL-9), and regulatory T cells (e.g., IL-10, TGF $\beta$, and FoxP3). Our study observed that placenta-derived MSCs undoubtedly exerted an anti-IL-5 effect in culture, and can therefore be considered a potential treatment for asthma.

In summary, our study results demonstrated that placenta-derived MSCs could suppress the proliferation of $\mathrm{CD} 4+\mathrm{T}$ cells and CD8+ $\mathrm{T}$ cells in all children and suppress the activation of CD4+ T cells and $\mathrm{CD} 8+\mathrm{T}$ cells in children with asthma. Placenta-derived MSCs exerted an anti-IL-5 effect and reduced IL-5 levels in an in vitro culture in different subgroups of the children with asthma. Notably, in the culture, MSCs at different concentrations exhibited the ability to suppress IL-5 in children with asthma. According to our study results, placenta-derived MSCs can be considered a potential cell-based therapy for asthma. Therefore, placenta-derived MSCs may be used in children with asthma who respond poorly to conventional treatments.

\section{Abbreviations}

Th2: type $2 \mathrm{~T}$ helper cell; Th1: type $1 \mathrm{~T}$ helper cell; IL: interleukin; MSCs: mesenchymal stem cells; iPSC-MSCs: induced pluripotent stem cell-derived MSCs; Treg: regulatory T; PBMCs: peripheral blood mononuclear cells; PBS: phosphate-buffered saline; ANOVA: one-way analysis of variance; TGF- $\beta$ : transforming growth factor-beta.

\section{Acknowledgments}

This work was supported by Taipei Medical University (TMU106-AE1-B01).

\section{Competing Interests}

The authors have declared that no competing interest exists.

\section{References}

1. Lin SC, Lin HW, Chiang BL. Association of croup with asthma in children: a cohort study. Medicine. 2017; 96(35):e7667.
2. Conese M, Piro D, Carbone A, Castellani S, Gioia SD. Hematopoietic and mesenchymal stem cells for the treatment of chronic respiratory diseases: role of plasticity and heterogeneity. The Scientific World Journal. 2014; 11: 1-11.

3. Parulekar AD, Atik MA, Hanania NA. Periostin, a novel biomarker of TH2-driven asthma. Curr Opin Pulm Med. 2014; 20: 60-5.

4. Bossios A, Sjöstrand M, Dahlborn AK, et al. IL-5 expression and release from human CD34 cells in vitro; ex vivo evidence from cases of asthma and Churg-Strauss syndrome. Allergy. 2010; 65: 831-9.

5. Skrindo I, Ballke C, Gran E, Johansen FE, Baekkevold ES, Jahnsen FL. IL-5 production by resident mucosal allergen-specific Tcells in an explant model of allergic rhinitis. Clin Exp Allergy. 2015; 45: 1296-304.

6. Buckley RH, Schiff SE, Schiff RI, et al. Hematopoietic Stem-Cell Transplantation for the Treatment of Severe Combined Immunodeficiency. N Engl J Med. 1999; 340:508-516.

7. Tan JW, Wang KY, Liao GJ, Chen F, Mu MZ. Neuroprotective effect of methylprednisolone combined with placenta-derived mesenchymal stem cell in rabbit model of spinal cord injury. Int J Clin Exp Pathol. 2015; 8:8976-82.

8. Grove JE, Bruscia E, Krause DS. Plasticity of bone marrow-derived stem cells. Stem Cells. 2004; 22: 487-500.

9. Lin HY, Xu L, Xie SS, et al. Mesenchymal stem cells suppress lung inflammation and airway remodeling in chronic asthma rat model via PI3K/Akt signaling pathway. Int J Clin Exp Pathol. 2015; 8:8958-67.

10. Inamdar AC, Inamdar AA. Mesenchymal stem cell therapy in lung disorders: pathogenesis of lung diseases and mechanism of action of mesenchymal stem cell. Exp Lung Res. 2013; 39: 315-27.

11. Bruno S, Camussi G. Role of mesenchymal stem cell-derived microvesicles in tissue repair. Pediatr Nephrol 2013; 28: 2249-54.

12. Lee JW, Fang X, Krasnodembskaya A, Howard JP, Matthay MA. Concise review: Mesenchymal stem cells for acute lung injury: role of paracrine soluble factors. Stem Cell. 2011; 29: 913-9.

13. Lazebnik LB, Trubitsyna IE, Agafonov MA, Kniazev OV, Liundup AV. Mesenchymal stromal cells transplantation in acute and chronic pancreatitis in rats. Eksp Klin Gastroenterol. 2011; (7): 28-31.

14. Park $\mathrm{CH}, \mathrm{Bae} \mathrm{SH}$, Kim HY, et al. A pilot study of autologous CD34-depleted bone marrow mononuclear cell transplantation via the hepatic artery in five patients with liver failure. Cytotherapy. 2013; 15: 1571-9.

15. Fu QL, Chow YY, Sun SJ, et al. Mesenchymal stem cells derived from human induced pluripotent stem cells modulate T-cell phenotypes in allergic rhinitis. Allergy. 2012; 67(10): 1215-22.

16. Desai MB, Gavrilova T, Liu J, et al. Pollen-induced antigen presentation by mesenchymal stem cells and $\mathrm{T}$ cells from allergic rhinitis. Clin Transl Immunology. 2013; 2(10):e7.

17. Bonfield TL, Koloze M, Lennon DP, Zuchowski B, Yang SE, Caplan AI. Human mesenchymal stem cells suppress chronic airway inflammation in the murine ovalbumin asthma model. Am J Physiol Lung Cell Mol Physiol. 2010; 299:L760-70

18. Goodwin $\mathrm{M}$, Sueblinvong V, Eisenhauer $\mathrm{P}$, et al. Bone marrow-derived mesenchymal stromal cells inhibit Th2- mediated allergic airways inflammation in mice. Stem Cells. 2011: 29:1137-48.

19. Kavanagh H, Mahon BP. Allogeneic mesenchymal stem cells prevent allergic airway inflammation by inducing murine regulatory T cells. Allergy. 2011; 66:523-31.

20. Lee SH, Jang AS, Kwon JH, Park SK, Won JH, Park CS. Mesenchymal stem cell transfer suppresses airway remodeling in a toluene diisocyanateinduced murine asthma model. Allergy Asthma Immunol Res. 2011; 3:205-11.

21. Park HK, Cho KS, Park HY, et al. Adipose- derived stromal cells inhibit allergic airway inflammation in mice. Stem Cells Dev. 2010; 19:1811-8.

22. Firinci F, Karaman M, Baran Y, et al. Mesenchymal stem cells ameliorate the histopathological changes in a murine model of chronic asthma. Int Immunopharmacol. 2011; 11:1120-6.

23. Nemeth K, Keane-Myers A, Brown JM, et al. Bone marrow stromal cells use TGF-beta to suppress allergic responses in a mouse model of ragweed-induced asthma. Proc Natl Acad Sci U S A. 2010; 107:5652-7.

24. Trzil JE, Masseau I, Webb TL, et al. Long-term evaluation of mesenchymal stem cell therapy in a feline model of chronic allergic asthma. Clin Exp Allergy. 2014; 44:1546-57.

25. Trzil JE, Masseau I, Webb TL, et al. Intravenous adipose-derived mesenchymal stem cell therapy. J Feline Med Surg. 2015; 17: 1-10.

26. Boulet LP, Fitzgerald JM, Levy ML, et al. Asthma guidelines implementation: a guide to the translation of GINA guidelines into improved care. Eur Respir J. 2012; 39: 1220-9.

27. Royce SG, Shen M, Patel KP, Huuskes BM, Ricardo SD, Samuel CS Mesenchymal stem cells and serelaxin synergistically abrogate established airway fibrosis in an experimental model of chronic allergic airways disease. Stem Cell Res. 2015; 25:495-505.

28. Lin SC, Huang JJ, Wang JY, Chuang HC, Chiang BL, Ye YL. Upregulated thymic stromal lymphopoietin receptor expression in children with asthma. Eur J Clin Invest. 2016; 46:511-9.

29. Su LJ, Wu M, Hui $\mathrm{Y}$, et al. Fluorescent nanodiamonds enable quantitative tracking of human mesenchymal stem cells in miniature pigs. Sci Rep. 2017; 7:45607.

30. Ge Q, Moir LM, Trian T, et al. The phosphoinositide 3'-kinase p110 delta modulates contractile protein production and IL- 6 release in human airway smooth muscle. J Cell Physiol. 2012; 227: 3044-52. 
31. Lin SC, Chou HC, Chen CM, Chiang BL. Anti-thymic stromal lymphopoietin antibody suppresses airway remodeling in asthma through reduction of MMP and CTGF. Pediatr Res 2019; 86:181-7.

32. Song X, Xie S, Lu K, Wang C. Mesenchymal Stem Cells Alleviate Experimental Asthma by Inducing Polarization of Alveolar Macrophages. Inflammation. 2015; 38: 485-92.

33. Kapoor S, Patel SA, Kartan S, Axelrod D, Capitle E, Rameshwar P. Tolerance-like mediated suppression by mesenchymal stem cells in patients with dust mite allergy-induced asthma. J Allergy Clin Immunol. 2012; 129:1094-101.

34. Leckie MJ. Anti-interleukin-5 monoclonal antibodies: preclinical and clinical evidence in asthma models. Am J Respir Med. 2003; 2:245-59.

35. Molfino NA, Gossage D, Kolbeck R, Parker JM, Geba GP. Molecular and clinical rationale for therapeutic targeting of interleukin-5 and its receptor. Clin Exp Allergy. 2012; 42:712-37.

36. Liu AH, Anderson WC 3rd, Dutmer CM, Searing DA, Szefler SJ. Advances in asthma 2015: Across the lifespan. J Allergy Clin Immunol. 2016; 138:397-404.

37. Nair P, Pizzichini MM, Kjarsgaard M, et al. Mepolizumab for prednisone dependent asthma with sputum eosinophilia. N Engl J Med. 2009; 360:985-93. 